\title{
OBESIDADE INFANTIL: TERAPIA NUTRICIONAL EM UM GRUPO DE USUÁRIOS DO SISTEMA ÚNICO DE SAÚDE - UM ESTUDO LONGITUDINAL
}

\section{CHILDHOOD OBESITY: NUTRITIONAL THERAPY IN A GROUP OF USERS OF THE UNIQUE HEALTH SYSTEM - A LONGITUDINAL STUDY}

\author{
Daniella Taborda ${ }^{1}$, Caryna Eurich Mazur ${ }^{2}$, Vania Schmitt ${ }^{3 *}$
}

\begin{abstract}
1 - Nutricionista. Especialista em Nutrição Clínica pelo Centro Universitário Campo Real, Guarapuava -PR.

2 - Nutricionista. Doutora em Medicina Interna - Universidade Federal do Paraná (UFPR). Docente da Universidade Estadual do Centro Oeste (UNICENTRO) - Guarapuava - PR, Brasil. 3 - Nutricionista. Docente do Centro Universitário Campo Real e Universidade Estadual do Centro Oeste. Doutoranda em Desenvolvimento Comunitário - Universidade Estadual do Centro Oeste (UNICENTRO) - Guarapuava - PR, Brasil.
\end{abstract}

\section{RESUMO:}

O excesso de peso em crianças e adultos brasileiros tem sido considerado um problema de saúde pública. O tratamento da obesidade infantil não é uma tarefa fácil, pois a mudança do comportamento alimentar representa um desafio para as redes de atenção à saúde, visto que ocorre apenas em médio e longo prazo e depende tanto de esforços individuais quanto do apoio de profissionais de saúde. O presente estudo objetivou identificar a efetividade na realização de terapia em grupo de crianças na faixa etária entre 06 a 12 anos diagnosticadas previamente com sobrepeso ou obesidade infantil no município de Guarapuava, Paraná, Brasil. Trata-se de um estudo longitudinal, com análise de dados provenientes dos prontuários de usuários atendidos entre os meses de março e dezembro de 2019. As informações foram obtidas por meio de coleta de dados em prontuário, obtendo dados de peso, estatura, índice de massa corporal para a idade, duração e tempo do tratamento. Participaram do grupo 10 crianças, sendo $60 \%$ do sexo masculino. Observouse que na maioria houve a manutenção do diagnóstico inicial, sendo sobrepeso ou obesidade, enquanto uma criança que se encontrava obesa foi classificada como sobrepeso após a intervenção. No entanto, três crianças apresentaram aumento no IMC, demonstrando ganho de peso proporcional ao crescimento ao considerar o estado anterior às intervenções. Conclui-se que a atuação do nutricionista e a adoção da estratégia de intervenção nutricional em grupos é importante, podendo auxiliar no tratamento de crianças com obesidade infantil, contribuindo assim, para a melhoria de sua qualidade de vida.

Palavras-chave: Atenção Primária à Saúde; Educação Alimentar e Nutricional; Saúde Pública.

\section{ABSTRACT:}

Overweight in Brazilian children and adults has been considered a public health problem. The treatment of childhood obesity is not an easy task, as the change in eating behavior represents a challenge for health care networks, as it occurs only in the medium and long term and depends both on individual efforts and on the support of health professionals. The present study aimed to identify the effectiveness in performing therapy in a group of children aged between 6 to 12 years previously diagnosed with overweight or childhood obesity in the city of Guarapuava, Paraná, Brasil. This is a longitudinal study, with analysis of data from the medical records of users attended between the months of March and December 2019. The information was obtained by collecting data from medical records, obtaining data 
on weight, height, index of body mass for age, duration and time of treatment. Ten children participated in the group, $60 \%$ of whom were male. It was observed that in the majority there was the maintenance of the initial diagnosis, being overweight or obese, while a child who was obese was classified as overweight after the intervention. However, three children showed an increase in BMI, showing weight gain proportional to growth when considering the state prior to the interventions. It is concluded that the role of the nutritionist and the adoption of a nutritional intervention strategy in groups is important, and may assist in the treatment of children with childhood obesity, thus contributing to the improvement of their quality of life.

Keywords: Primary Health Care; Food and Nutrition Education; Public Health.

\section{INTRODUÇÃO}

O excesso de peso entre crianças brasileiras tem sido considerado um problema de saúde pública, sabe-se que nas últimas décadas do século XX houve grande aumento da substituição do problema de escassez de alimentos pelo de excesso. Entre as principais etiologias da obesidade infantil está o acréscimo da densidade energética alimentar, onde as crianças estão aumentando o consumo de produtos pobres em nutrientes e com conteúdo elevado em açúcar (BOMFIM et al., 2016; HENRIQUES et al., 2018). A obesidade está condicionada a fatores biológicos, ambientais, socioeconômicos, demográficos, psicossociais, culturais, de alimentação, sedentarismo, como se verifica entre as crianças e os adolescentes que permanecem muitas horas sentadas em frente à televisão, videogames e computadores, muitas vezes acompanhados de produtos ultra processados, geralmente com alto teor calórico (HENRIQUES et al., 2018).

Assim, por ser uma doença de etiologia multifatorial, também envolve a interação de influências metabólicas, fisiológicas, comportamentais, além disso, a obesidade está associada com aumento de risco para hipertensão arterial sistêmica, anormalidades lipídicas, diabetes mellitus, doença coronariana (sendo diagnosticada em aproximadamente $40 \%$ das crianças obesas), disfunção endotelial precoce em crianças e adolescentes obesos, sendo importantes preocupações em saúde pública, gerando a frustração para profissionais da saúde e pacientes e, sobretudo, um grande desafio terapêutico (MELLO et al., 2004). Além dos fatores mencionados, muitas vezes a obesidade acarreta dificuldades comportamentais, interferindo assim, no relacionamento social, familiar e o desenvolvimento da depressão infantil (TENORIO et al., 2011).

A introdução inadequada de alimentos e o desmame precoce já no primeiro ano de vida podem levar ao início da fisiopatologia da obesidade em indivíduos predispostos. E 
como uma forma de minimizar tal condição, existe a recomendação de que a regulamentação para comercialização de alimentos e bebidas complementares sejam mais rigorosas no sentido de limitar o consumo de produtos com alto teor de gordura, açúcar e sal por esse público. Além de orientar os cuidadores que estimulem o consumo variado de alimentos saudáveis. Ainda se fazem necessárias recomendações destinadas a melhorar os índices de amamentação (KOPLIN et al. 2019; LOURENÇO et al., 2018).

Das crianças menores de dois anos de idade, quase um terço já consome refrigerantes e sucos artificiais contendo açúcar, e mais de 60\% comem biscoitos e bolos. As práticas alimentares estabelecidas na infância tendem a sustentar-se na vida adulta (HENRIQUES et al., 2018).

No entanto, o tratamento da obesidade infantil não é uma tarefa fácil. A mudança do comportamento alimentar representa um desafio para os sistemas de saúde, pois ocorre apenas em médio e longo prazos e depende tanto de esforços individuais quanto do apoio de profissionais de saúde. Nesse contexto, o enfrentamento da obesidade infantil deve ser pautado em políticas públicas e estratégias voltadas para a educação alimentar e nutricional (MELLO et al., 2004).

A prevenção da obesidade pode se iniciar desde idades precoces a fim de reverter o aumento acelerado dessa doença, no entanto, estudos de intervenção da obesidade infantil em longo prazo, incluindo mudanças de comportamento e com tamanho amostral suficiente no Brasil, ainda são escassos (POETA et al., 2013). A terapia nutricional em grupo pode ser uma estratégia utilizada para o tratamento da obesidade infantil por meio da educação alimentar e nutricional. Assim, pode-se aplicar diversas formas para incentivar, facilitar e apoiar a prática de novos hábitos adequados e saudáveis, possivelmente melhorando a qualidade de vida e facilitando a adesão ao tratamento.

Dessa forma, o presente estudo objetivou propiciar a mudança de comportamentos dos indivíduos participantes, por meio da promoção da alimentação adequada e saudável pela realização de terapia em grupo de crianças na faixa etária entre 06 a 12 anos diagnosticadas previamente com sobrepeso ou obesidade infantil no município de Guarapuava-PR. 


\section{MATERIAL E MÉTODOS}

Caracteriza-se como um estudo longitudinal retrospectivo, com análise de dados provenientes dos prontuários das crianças atendidas entre os meses de março e dezembro de 2019 em uma Unidade Básica de Saúde no município de Guarapuava-PR. O estudo foi aprovado pelo comitê de ética em pesquisa do Centro Universitário Campo Real sob parecer número 4.033.417/2020.

Foram coletados dados dos prontuários de crianças, sendo meninas e meninos com idade entre 6 e 12 anos participantes das atividades do grupo de intervenção e terapia nutricional para crianças obesas de uma Unidade Básica de Saúde. Para o estudo foram utilizados dados iniciais de peso, estatura e índice de massa corporal. Também, os dados intermediários e finais possibilitando análise dos resultados obtidos. O IMC para a idade foi o critério utilizado para classificar o estado nutricional das crianças, seguindo os parâmetros propostos pela Organização Mundial da Saúde (WHO, 2006; 2007). Para complementar a análise, foram coletados dados referentes ao sexo, peso, estatura em cada encontro do grupo.

No decorrer do período selecionado para a pesquisa algumas crianças procuraram outros tratamentos de saúde no local e após serem diagnosticadas com obesidade foram encaminhadas para tratamento nutricional e inserção no grupo de estudo. Isso justifica o fato de que algumas crianças participaram de menos encontros que outras, ou seja, o grupo foi incorporando crianças ao longo do ano conforme demanda.

Os encontros foram realizados mensalmente por meio de roda de conversa, o que proporcionava a interação dos pais e crianças pela utilização de atividades práticas e teóricas que contavam com a participação de outros profissionais como: dentista, enfermeira, fisioterapeuta e seus estagiários, estagiária de psicologia e de nutrição. Os temas abordados e as estratégias utilizadas em cada encontro podem ser observados no Quadro 1. 
Quadro 1 - Temas abordados e estratégias utilizadas em grupo de intervenção e terapia nutricional para crianças obesas.

\begin{tabular}{|c|c|c|}
\hline Mês & Tema Abordado & $\begin{array}{c}\text { Estratégia Utilizada } \\
\end{array}$ \\
\hline Março & $\begin{array}{l}\text { Apresentação do grupo e } \\
\text { entrega do plano alimentar }\end{array}$ & $\begin{array}{l}\text { Apresentação dos objetivos do tratamento nutricional em grupo } \\
\text { aos pais e às crianças. Orientação de como realizar o plano } \\
\text { alimentar e a apresentação do painel de metas representado } \\
\text { por estrelinhas que serão afixadas em cada encontro conforme } \\
\text { o cumprimento das metas apresentadas aos pais e as crianças, } \\
\text { como estímulo ao tratamento. Entrega de uma carteirinha de } \\
\text { acompanhamento do desenvolvimento antropométrico } \\
\text { personalizada para cada criança. }\end{array}$ \\
\hline Abril & $\begin{array}{l}\text { Redução do consumo de } \\
\text { açúcar }\end{array}$ & $\begin{array}{l}\text { Demonstração da quantidade de açúcar presente nos } \\
\text { alimentos, seus malefícios quando consumidos em excesso e } \\
\text { análise dos rótulos. }\end{array}$ \\
\hline Maio & $\begin{array}{l}\text { Estimulando o consumo } \\
\text { de frutas e verduras }\end{array}$ & $\begin{array}{l}\text { As crianças com os olhos fechados retiravam os alimentos de } \\
\text { uma caixa personalizada e através do tato identificavam o } \\
\text { alimento abordando seu valor nutricional. }\end{array}$ \\
\hline Junho & $\begin{array}{l}\text { Prato saudável, } \\
\text { estimulando a autonomia } \\
\text { das crianças }\end{array}$ & $\begin{array}{l}\text { Apresentação da pirâmide dos alimentos. As crianças foram } \\
\text { divididas em dois grupos, sendo um responsável pela } \\
\text { elaboração do prato saudável do café da manhã e o outro do } \\
\text { almoço, utilizando os alimentos da pirâmide alimentar. }\end{array}$ \\
\hline Julho & $\begin{array}{l}\text { Cumprimento de metas } \\
\text { nas férias }\end{array}$ & $\begin{array}{l}\text { Conversa sobre a importância dos hábitos alimentares em } \\
\text { todos os períodos do ano, principalmente nas férias. Bem como } \\
\text { sobre os benefícios do envolvimento das crianças na cozinha e } \\
\text { em outras atividades envolvendo alimentação. }\end{array}$ \\
\hline Agosto & $\begin{array}{l}\text { Degustação de olhos } \\
\text { vendados }\end{array}$ & $\begin{array}{l}\text { As crianças tiveram seus olhos vendados. Em seguida foram } \\
\text { oferecidos alguns alimentos às crianças para que fossem } \\
\text { degustados e identificados o seu sabor. Em seguida foram } \\
\text { entreguem imagens desses alimentos para que elas colorissem } \\
\text { o alimento que mais gostou e aquele que menos gostou. }\end{array}$ \\
\hline Setembro & Cultivando minha horta & $\begin{array}{l}\text { Foram abordadas sobre diferentes formas de como cultivar uma } \\
\text { horta. Em seguida foi entregue uma muda de alface para que } \\
\text { cada criança plantasse em sua casa com o auxílio dos pais. }\end{array}$ \\
\hline Outubro & $\begin{array}{l}\text { Importância da mastigação } \\
\text { e percepção dos sabores }\end{array}$ & $\begin{array}{l}\text { As crianças foram vendadas e em seguida oferecidos a elas } \\
\text { alimentos: azedo (limão), doce (chocolate ao leite), amargo } \\
\text { (rúcula), umami (tomate cereja) e salgado (bolacha água e sal) } \\
\text { para que identificassem o sabor e quantas vezes foi preciso } \\
\text { mastigar aquele alimento. }\end{array}$ \\
\hline Novembro & $\begin{array}{l}\text { Estímulo do consumo de } \\
\text { água e a importância da } \\
\text { atividade física } \\
\text { diariamente }\end{array}$ & $\begin{array}{l}\text { Circuito de exercícios físicos elaborados pelos estagiários de } \\
\text { fisioterapia. Entrega de garrafas plásticas para cada criança a } \\
\text { fim de estimular o consumo diário de água. Roda de conversa } \\
\text { com interação dos pais e crianças. }\end{array}$ \\
\hline Dezembro & $\begin{array}{l}\text { Encerramento do grupo e } \\
\text { demonstração dos } \\
\text { resultados }\end{array}$ & $\begin{array}{l}\text { Confraternização para o encerramento das atividades e } \\
\text { discussão dos resultados obtidos. }\end{array}$ \\
\hline
\end{tabular}

Os resultados foram avaliados por meio de estatística descritiva com utilização de valores absolutos no software Microsoft Excelß versão 2017 para Windows.

\section{RESULTADOS}

Foram coletados dados das 10 crianças com idade entre 6 e 12 anos participantes 
do grupo de intervenção e terapia nutricional para crianças obesas. Os dados iniciais e finais do acompanhamento realizado com as crianças podem ser observados na Tabela 1, sendo possível perceber a maior prevalência do sexo masculino $(60 \% ; n=6)$.

Tabela 1 - Dados descritivos iniciais e finais de acompanhamento de crianças obesas em grupo de intervenção e terapia nutricional.

\begin{tabular}{lccccccc}
\hline & \multicolumn{3}{c}{ Dados iniciais } & \multicolumn{3}{c}{ Dados finais } \\
\hline Sexo & $\begin{array}{c}\text { Idade } \\
(\mathbf{a n o s})\end{array}$ & $\begin{array}{c}\text { Peso } \\
(\mathbf{k g})\end{array}$ & $\begin{array}{c}\text { Altura } \\
(\boldsymbol{m})\end{array}$ & $\begin{array}{c}\text { IMC/idade } \\
\left(\mathbf{k g} / \mathbf{m}^{2}\right)\end{array}$ & $\begin{array}{c}\text { Peso } \\
(\mathbf{k g})\end{array}$ & $\begin{array}{c}\text { Altura } \\
(\boldsymbol{m})\end{array}$ & $\begin{array}{c}\text { IMC } \\
\left(\mathbf{k g} / \mathbf{m}^{2}\right)\end{array}$ \\
\hline \multirow{4}{*}{ Feminino } & 9 & 41,7 & 1,39 & 21,58 & 45,2 & 1,45 & 21,50 \\
& 10 & 67,1 & 1,58 & 26,90 & 65,7 & 1,59 & 26,00 \\
& 10 & 43,8 & 1,43 & 21,42 & 47,1 & 1,46 & 22,09 \\
& 11 & 72,0 & 1,54 & 30,40 & 73,3 & 1,59 & 29,00 \\
\hline \multirow{4}{*}{ Masculino } & 6 & 27,1 & 1,19 & 19,14 & 32,0 & 1,24 & 20,81 \\
& 7 & 26,7 & 1,20 & 18,54 & 25,5 & 1,22 & 17,13 \\
& 9 & 39,0 & 1,33 & 22,05 & 42,3 & 1,36 & 22,90 \\
& 9 & 55,4 & 1,45 & 26,35 & 55,3 & 1,50 & 24,58 \\
& 10 & 65,8 & 1,50 & 29,24 & 67,3 & 1,54 & 28,32 \\
& 11 & 68,0 & 1,63 & 25,60 & 64,1 & 1,68 & 22,71 \\
\hline
\end{tabular}

A Tabela 2 demonstra dados referentes às diferenças de peso, altura e classificação do IMC para a idade inicial e final, após o término do período de intervenção. Bem como, por quanto tempo as crianças participaram do grupo. É possível observar que duas crianças que se encontravam em sobrepeso no início do acompanhamento mantiveram essa classificação, enquanto uma criança que se encontrava obesa foi classificada como sobrepeso após a intervenção.

Apesar da manutenção da obesidade na maioria das crianças, apenas três tiveram aumento no IMC após o período de intervenção, demonstrando que o ganho de peso foi proporcional ao crescimento, considerando o estado anterior às intervenções.

Com relação ao peso, oito crianças apresentaram aumento, enquanto em duas 
houve redução. Sobre a altura, todas as crianças obtiveram aumento, sendo no mínimo 0,01m e no máximo $0,05 \mathrm{~m}$, ou seja, 1 e 5 centímetros.

Quanto à duração do tratamento, apenas duas crianças participaram dos dez encontros. O menor período de participação foi de três meses. A baixa adesão se deu principalmente pelo estudo ser realizado em uma unidade de saúde pequena e a população depender de apoio de seus pais ou responsáveis para a participação, principalmente na questão de transporte até o local das reuniões.

Tabela 2 - Análise do acompanhamento de crianças obesas em grupo de intervenção e terapia nutricional.

\begin{tabular}{|c|c|c|c|c|c|}
\hline & $\begin{array}{c}\text { Diferença } \\
\text { de peso } \\
\text { (em } \mathrm{kg})\end{array}$ & $\begin{array}{c}\text { Diferença de } \\
\text { altura } \\
(\mathrm{em} \mathrm{m})\end{array}$ & $\begin{array}{l}\text { IMC/idade } \\
\text { inicial } \\
\text { (classificação) }\end{array}$ & $\begin{array}{l}\text { IMC/idade } \\
\text { final } \\
\text { (classificação) }\end{array}$ & $\begin{array}{l}\text { Tempo de } \\
\text { tratamento } \\
\text { (em meses) }\end{array}$ \\
\hline \multirow{4}{*}{ Feminino } & $+3,5$ & 0,05 & Obesidade & Obesidade & 9 \\
\hline & $+1,4$ & 0,01 & Obesidade & Obesidade & 3 \\
\hline & $+3,3$ & 0,03 & Sobrepeso & Sobrepeso & 4 \\
\hline & $+1,3$ & 0,05 & Obesidade & Obesidade & 5 \\
\hline \multirow{6}{*}{ Masculino } & $+4,9$ & 0,05 & Obesidade & Obesidade & 10 \\
\hline & $-1,2$ & 0,02 & Sobrepeso & Sobrepeso & 7 \\
\hline & $+3,3$ & 0,03 & Obesidade & Obesidade & 10 \\
\hline & $-0,1$ & 0,05 & Obesidade & Obesidade & 6 \\
\hline & $+1,5$ & 0,04 & Obesidade & Obesidade & 6 \\
\hline & $+3,9$ & 0,05 & Obesidade & Sobrepeso & 5 \\
\hline
\end{tabular}

Visando maior compreensão sobre o acompanhamento que as crianças receberam no grupo de intervenção, o Quadro 1 demonstra os temas abordados e as estratégias utilizadas em cada um dos dez encontros.

\section{DISCUSSÃO}

No intuito de auxiliar as crianças no tratamento durante os encontros foram abordados 
diferentes temas desde a apresentação dos objetivos do tratamento nutricional em grupo aos pais e às crianças, os malefícios de uma alimentação incorreta, o valor nutricional da alimentação, dentre outros temas. O principal objetivo almejado foi propiciar a mudança de comportamentos dos indivíduos participantes, por meio da promoção da alimentação adequada e saudável, sendo essa uma das diretrizes da Política Nacional de Alimentação e Nutrição e que pode contribuir para segurança alimentar e nutricional da população e o alcance do Direito Humano à Alimentação Adequada e Saudável (GOMES; PINTO, 2013).

Em consonância com o Guia Alimentar para a População Brasileira (BRASIL, 2014), para uma melhora nos hábitos alimentares e avanços na qualidade de vida, no grupo foram abordados temas que propiciaram o conhecimento da pirâmide dos alimentos, a importância de escolher alimentos mais saudáveis, bem como o aumento do consumo diário de água e a realização de exercícios físicos, visando avanços no estudo e o desenvolvimento de hábitos alimentares saudáveis.

Sobre o ganho de peso observado na amostra, é importante ressaltar que na infância e adolescência ocorrem inúmeras mudanças físicas e psicológicas, as crianças estão na fase de crescimento e o ganho de peso é normal devido ao desenvolvimento fisiológico individual. Gomes e Pinto (2013), afirmam que elas são influenciadas por um ambiente social onde comida pouco saudável e comodidades da vida moderna, como telefones celulares e videogames, predominam. Porém, para que tenham um crescimento saudável necessitam ser influenciadas pelos pais e o meio que as cercam de maneira correta.

Dois dos dez participantes do grupo mantiveram o IMC classificado como sobrepeso ao início e término do tratamento e um participante conseguiu ao término do tratamento sair do quadro de obesidade para sobrepeso. A não alteração na classificação do IMC pode ter ocorrido por alguns não seguirem as recomendações em casa ou nas férias escolares, outros por não fazer exercícios físicos aliados a dieta. Porém, todos cresceram em estatura e estão em fase de crescimento ganhando também peso corporal. Enes (2010) ao estudar a intervenção em grupo relata que a variável do IMC, sofre alteração significativa e eficiente em pacientes que utilizaram atividade física ou exercício como método de auxílio no tratamento da obesidade infantil/adolescente. Dessa forma, é importante ressaltar o papel da família durante o processo, uma vez que o incentivo dos pais ou responsáveis é fundamental para o alcance dos objetivos.

Como já mencionado, a maioria dos avaliados mantiveram a classificação IMC inicial mesmo após o aumento do peso corporal, isso se deve ao fato que todos estão ainda 
na fase de crescimento, logo, cresceram em altura mantendo então no cálculo do IMC valores semelhantes. Enes (2010) concorda que é necessário considerar o aspecto do processo de maturação biológica, pois se tratando de um grupo de crianças, existe o fato delas estarem em fase de crescimento e alguns resultados podem ser mascarados devido a essa questão.

Algumas crianças faltaram encontros e foi possível perceber que após o início das férias letivas muitos desistiram. Com isso é possível sugerir a grande influência dos pais no tratamento dos filhos, visto que Gomes e Pinto (2013) concordam e relatam em seus estudos que os pais têm um grande poder de influenciar seus filhos, desde a ter uma alimentação adequada e saudável como também à prática de atividades físicas, porém, também podem ser maus influenciadores.

Durante o estudo foi possível perceber o baixo número de interessados, bem como também algumas desistências antes do término do cronograma, isso se deve ao fato de as crianças dependerem dos pais ou responsável para comparecer ao grupo. Ao considerar o estudo de Sichieri e Souza (2008) é visto que os programas de prevenção da obesidade para crianças mostram que as intervenções que produziram maiores efeitos incluíram a participação dos pais, incentivando os filhos a se obter uma mudança no estilo de vida alimentar.

Para estudos futuros relacionados ao tema sugere-se reuniões com os pais em separado dos filhos, mostrando por meio de dados científicos e estudos já realizados a importância do apoio familiar na mudança dos hábitos alimentares. Para as crianças às estratégias recomendadas são: mostrar por meio de filmes ou vídeos os malefícios que uma má alimentação pode causar no decorrer da vida adulta; desenvolver jogos que estimulem o interesse dos participantes e o espírito competitivo melhorando assim também a adesão.

Dentre as limitações encontradas as que mais sobressaíram estão relacionadas à baixa adesão das crianças e pais ao grupo, às faltas das crianças devido ao período de férias escolares e a disponibilidade dos pais e ou responsáveis por levar as crianças até o grupo na unidade de saúde.

\section{CONCLUSÃO}

Após análise dos dados resultantes do grupo de intervenção nutricional com crianças, percebe-se que apesar do ganho de peso apresentado por parte das crianças, 
houve crescimento em estatura. Dessa forma, apesar da maioria das crianças manter a classificação do estado nutricional em obesidade, percebe-se que o ganho de peso foi proporcional ao crescimento, considerando o estado anterior às intervenções, logo denotase a importância do acompanhamento nutricional em toda a infância.

Em resumo, esse trabalho mostra a importância da atuação do nutricionista e da estratégia de intervenção nutricional em grupos. Tais intervenções podem auxiliar no tratamento de crianças com obesidade infantil, para que eles possam se alimentar de forma adequada, atingindo as recomendações diárias de todos os nutrientes e reduzindo as chances de se tornar um adulto obeso e com possibilidade de desenvolvimento de doenças.

\section{REFERÊNCIAS}

ARAÚJO, Rafael André; BRITO, Ahécio Araujo; SILVA, Francisco Martins. O papel da educação física escolar diante da epidemia da obesidade em crianças e adolescentes. Educação física em revista. [online] ISSN: 1983-6643 Vol.4 №2 mai/jun/jul/ago - 2010. Brasília, 2010. Disponível em:<http://portalrevistas.ucb.br/index.php/efr/article/viewArticle/1651>. Acesso em 22/04/2020.

BOMFIM, Natália da Silva; GUILHERME, Cristiane Silva. SAITO, Jéssica de Almeida; MONTEZANI, Érica. Obesidade infantil: principais causas e a importância da intervenção nutricional. Revista Científica da escola da Saúde. Ano 5, n 1, p. 31-44, out.2015/jan.2016. Rio Grande do Norte, 2016. Disponível em: < file://C:/Users/op/Downloads/1243Texto\%20do\%20artigo-5693-1-1020160921.pdf>. Acesso em: 22/04/2020.

BRASIL. Ministério da Saúde. Secretaria de Atenção Primaria à Saúde. Departamento de Promoção da Saúde. Guia alimentar para crianças brasileiras menores de 2 anos / Ministério da Saúde, Secretaria de Atenção Primaria à Saúde, Departamento de Promoção da Saúde. - Brasília: Ministério da Saúde, 2019.

ENES, Carla Cristina; SLATER, Betzabeth. Obesidade na adolescência e seus principais fatores determinantes. Departamento de Nutrição da Faculdade de Saúde Pública da Universidade de São Paulo. Revista Brasileira Epidemiológica. 2010; 13(1): p. 163-71. São Paulo, 2010.

GOMES, Ana Lidia Garcia; PINTO, Ricardo Figueiredo. OBESIDADE INFANTIL: Uns quilos 
a mais hoje, uns anos a menos no futuro.2013. 19f. Artigo de Conclusão de Curso (Graduação em Educação Física), Universidade do Estado do Pará, 2013.

HENRIQUES, Patrícia; O'DWYER, Gisele; DIAS, Patrícia Camacho; BARBOSA, Roseane Moreira Sampaio; BURLANDY, Luciene. Políticas de Saúde e de Segurança Alimentar e Nutricional: desafios para o controle da obesidade infantil. Ciência e saúde coletiva [online]. 2018, vol.23, n.12, pp.4143-4152. ISSN 1678-4561. Rio de Janeiro, Dez. 2018. Disponível em: < https://doi.org/10.1590/1413-812320182312.34972016. >. Acesso em 08/04/2020.

LOURENÇO, Adriana de Sousa Nagahashi; NERI, Daniela Almeida; KONSTANTYNER, Tulio; PALMA, Domingos; OLIVEIRA, Fernanda Luisa Ceragioli. Fatores associados ao ganho de peso rápido em pré-escolares frequentadores de creches públicas. Revista paulista de pediatria. [online]. 2018, vol.36, n.3, pp.292-300. ISSN 1984-0462. São Paulo, set. 2018. Disponível em: <https://doi.org/10.1590/1984-0462/;2018;36;3;00012>. Acesso em 08/04/2020.

MELLO, Elza; LUFT, Vivian; MEYER, Flávia. Atendimento ambulatorial individualizado programa de educação em grupo: qual oferece mais mudança de hábitos alimentares e de atividade física em crianças obesas? Jornal de Pediatria. Rio de Janeiro [online]. 2004, vol.80, n.6, pp.468-474. ISSN 1678-4782. Disponível em: < https://doi.org/10.1590/S002175572004000800008>. Acesso em 05/05/2020.

PLANO NACIONAL DA PRIMEIRA INFÂNCIA. Mapeamento da Ação Finalística "Criança com Saúde". Obesidade na primeira infância. Rede Nacional Primeira Infância - RNPI. 80p. set. 2014. Fortaleza, 2014. Disponível em: <http://primeirainfancia.org.br/>. Acesso em: 03/05/2020.

POETA, Lisiane Schilling; DUARTE, Maria de Fátima da Silva; CARAMELLI, Bruno; MOTA, Jorge; GIULIANO, Isabela de Carlos Back. Efeitos do exercício físico e da orientação nutricional no perfil de risco cardiovascular de crianças obesas. Revista da Associação Médica Brasileira. [online]. 2013, vol.59, n.1, pp.56-63. ISSN 0104-4230. Florianópolis SC, 2013. Disponível em: <https://doi.org/10.1590/S0104-42302013000100012>. Acesso em: 15/05/2020.

SICHIERI, Rosely; SOUZA, Rita Adriana de. Estratégias para prevenção da obesidade em 
crianças e adolescentes. Cadernos de Saúde Pública [online], ISSN 1678-4464. v.24, supl.2. Rio de Janeiro, 2008. Disponível em: <https://doi.org/10.1590/S0102311X2008001400002>. Acesso em: 03/05/2020.

TENORIO, Aline e Silva; COBAYASHI, Fernanda. Obesidade infantil na percepção dos pais. Revista paulista de pediatria. vol.29 no.4 São Paulo Dec. 2011. Disponível em: <http://dx.doi.org/10.1590/S0103-05822011000400025>. Acesso em: 15/05/2020.

WHO - World Health Organization. Child Growth Standards. Department of Nutrition for Health and Development. Geneva, 2006-2007. OMS - Organização Mundial de Saúde. Padrões de crescimento infantil. Disponível em: <https://www.who.int/childgrowth/standars/ Technical_report.pdf>. Acesso em: 01/05/2020.

\author{
*Autor para correspondência: \\ Vania Schmitt \\ Universidade Estadual do Centro Oeste \\ R. Salvatore Renna, 875 - Santa Cruz, Guarapuava - PR, 85015-430 \\ Email: vania_schmitt@hotmail.com \\ Recebido: 01/08/2020 Aceite: 24/09/2020
}

